SCIENTIFIC REPORT

\title{
Ultrasound biomicroscopy: role in diagnosis and management in 130 consecutive patients evaluated for anterior segment tumours
}

\author{
R M Conway, T Chew, P Golchet, K Desai, S Lin, J O’Brien
}

Br J Ophthalmol 2005;89:950-955. doi: 10.1136/bjo.2004.059535

Background/aim: Ultrasound biomicroscopy (UBM) is an important tool for assessing anterior segment pathology. This study sought to evaluate UBM in the management of anterior segment fumours.

Methods: Retrospective analysis of medical records of consecutive patients referred to the ocular oncology unit, University of California San Francisco (UCSF), for suspected anterior segment tumours from 1999 to 2004.

Results: 132 eyes from 130 patients were evaluated, including 55 uveal melanomas (UM), 21 iris naevi, 30 iris cysts, and 26 remaining lesions. Of the melanomas, 45 were also evaluated with conventional A/B-scan. There was $29 \%$ correspondence between the anatomical structures invaded by melanoma as identified by B-scan $v$ disease extent defined by UBM. Ciliary body and peripheral iris involvement by melanomas was significantly more frequently observed by UBM than B-scan. Seven of 30 benign cysts were diagnosed as cystic before UBM evaluation. In three cases, neuroepithelial cysts were associated with intercurrent pathology including iris naevus $(n=2)$ and ciliary body melanoma $(n=1)$. Two ciliary body melanomas showed cavitation, including one patient with a pseudocyst. Histopathological correlation was possible in six cases.

Conclusion: UBM is an indispensable tool for the management of anterior segment tumours. This study demonstrates the superiority of UBM $v$ conventional B-scan for the precise localisation of uveal melanoma, especially involving the ciliary body and peripheral iris.

$\mathrm{U}$ ltrasound biomicroscopy (UBM) has dramatically improved resolution of anterior segment tumours, from 300-400 $\mu \mathrm{m}$ with conventional $10 \mathrm{MHz}$ ultrasonography down to $20-50 \mu \mathrm{m}$ with tissue penetration of up to $4 \mathrm{~mm} .{ }^{1}$ This technique allows for in vivo high resolution imaging of anterior segment masses with biometry and assessment of adjacent tissue invasion. ${ }^{1-4}$ Recent series have demonstrated a high correlation between UBM features of anterior uveal melanomas (UM) and histopathological features, including local extension. ${ }^{4}$ Historically biopsy, resection, or enucleation were the main options for diagnosis or treatment of anterior segment malignancy. In many cases, UBM allows for definitive diagnosis and more conservative management, with comparable or better outcomes. $^{2}$

We describe our experience with UBM in managing 130 patients referred to an ocular oncology centre for evaluation of suspected anterior segment tumours.

\section{METHODS}

Medical records of consecutive patients referred to the University of California San Francisco ocular oncology unit between January 1999 and July 2004 for evaluation of anterior segment tumours were reviewed. Patients underwent a complete ophthalmic evaluation including gonioscopy, slit lamp photography, and iris fluorescein angiography. Where clinically indicated, A/B-scan ultrasonography was performed in selected patients. A/B ultrasounds were evaluated by two ophthalmologists (JO/RMC) experienced in their interpretation.

UBM was carried out utilising a $50 \mathrm{MHz}$ commercially available device (Zeiss Humphrey, San Leandro, CA, USA) using an eye cup with methylcellulose medium. UBM examinations were performed by one ophthalmologist with extensive UBM experience (SL). Both maximal tumour thickness and maximal tumour diameter were documented on each occasion by multiple radial and cross sectional measurements.

To compare A/B-scan ultrasound with UBM, observers were blinded to the patients' data. Two observers experienced in $\mathrm{A} / \mathrm{B}$-scan and UBM interpretation independently performed the analysis (RMC/JO). Features assessed included the anatomical structures invaded by tumour. Fisher's exact test was used to test for statistically significant associations between unpaired data.

\section{RESULTS}

A total of 130 patients were identified (table 1). Seventy per cent of lesions were referred as melanomas, but demonstrated neuroepithelial cysts on UBM. In $93 \%$ of these, UBM was required to make the diagnosis (table 2, fig 1). Neuroepithelial cysts coexisted with naevi $(\mathrm{n}=2)$ and ciliary body (CB) melanoma ( $\mathrm{n}=1$ ) (fig 1 ).

Thirty nine solid iris lesions were initially observed (mean follow up 28 months, range 9-58 months) by serial photography and UBM biometry. Eighteen showed growth and were reclassified as melanoma and underwent proton beam radiotherapy (PBT) (table 2, fig 2). Additionally, all had one or more other features of malignancy. ${ }^{5} \mathrm{CB}$ invasion was detected in 14 cases (table 3, fig 2). The remaining 21 cases showed no growth by UBM (mean follow up 26 months) and were characterised as naevi.

Sixteen CB melanomas were identified (table 2, figs 1 and $3)^{5}$; 12 presented with visual loss secondary to lens subluxation, cataract, glaucoma, or retinal detachment. Extrascleral extension $(\mathrm{n}=1)$ and ring configuration $(\mathrm{n}=1)$ were also encountered. Iris invasion was observed by UBM in eight patients (table 3). CB melanomas were managed by enucleation or PBT. In all enucleated specimens

Abbreviations: $C B$, ciliary body; $P B T$, proton beam radiotherapy; UBM, ultrasound biomicroscopy; UCSF, University of California San Francisco; UM, uveal melanomas 

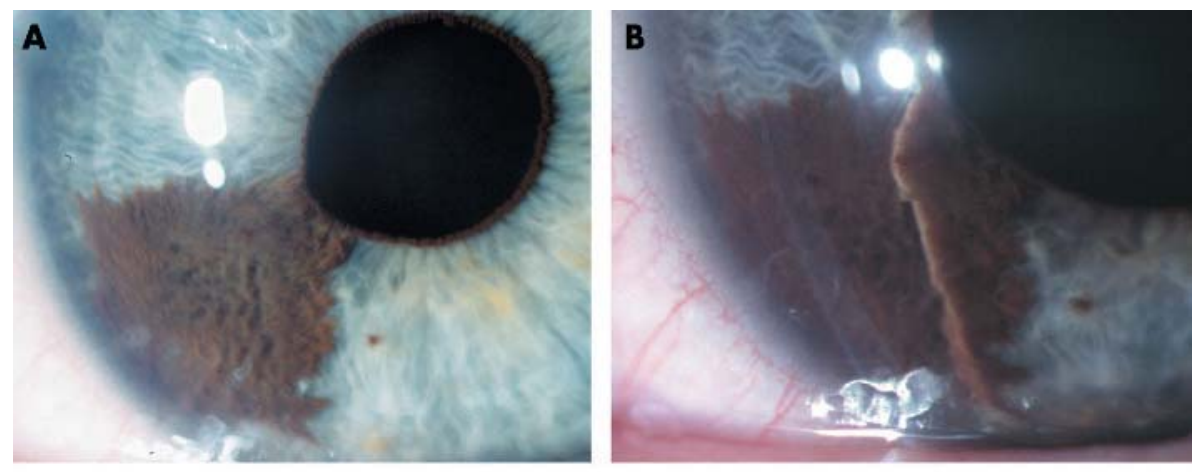

Figure 1 Neuroepithelial cyst and naevus associated with cyst. (A) Slit lamp photograph, right eye, of pigmented iris naevus. Note mild pupil distortion. (B) Higher power showing elevation of the lesion in (A). (C) UBM of the naevus in (A) and (B) demonstrates an associated thin walled

neuroepithelial cyst (c, cyst, n, naevus). (D) UBM of a typical peripheral neuroepithelial cyst with a thin cyst wall and anechoic centre (c, cyst). Note angle closure is present in the region of the cyst. (E) Slit lamp photograph, left eye, of a ciliary body melanoma (*) with an associated large neuroepithelial
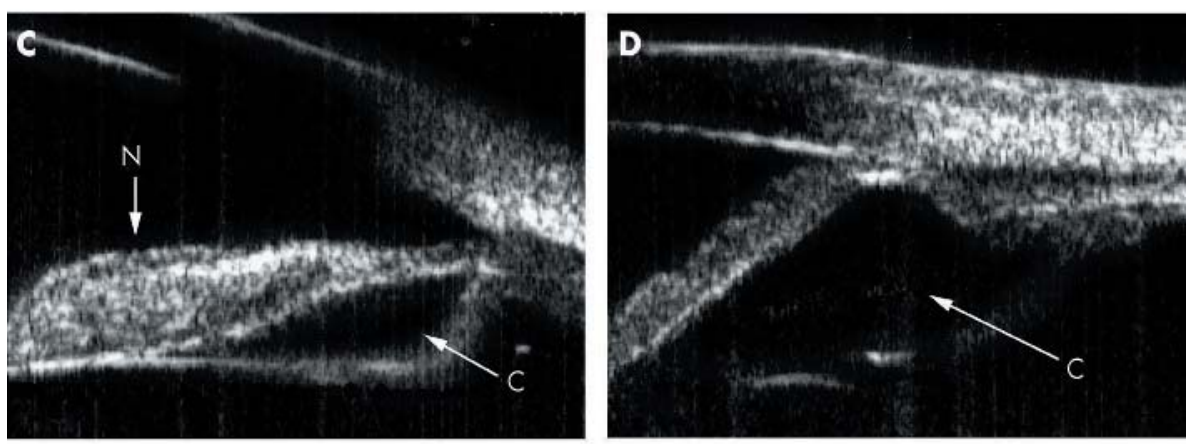
cyst. The cyst is visible at the pupil (arrow). (F) UBM of the patient in (E). A large cyst with features resembling a primary neuroepithelial cyst including thin, highly reflective cyst wall (arrows) is associated with the ciliary body melanoma (not shown).
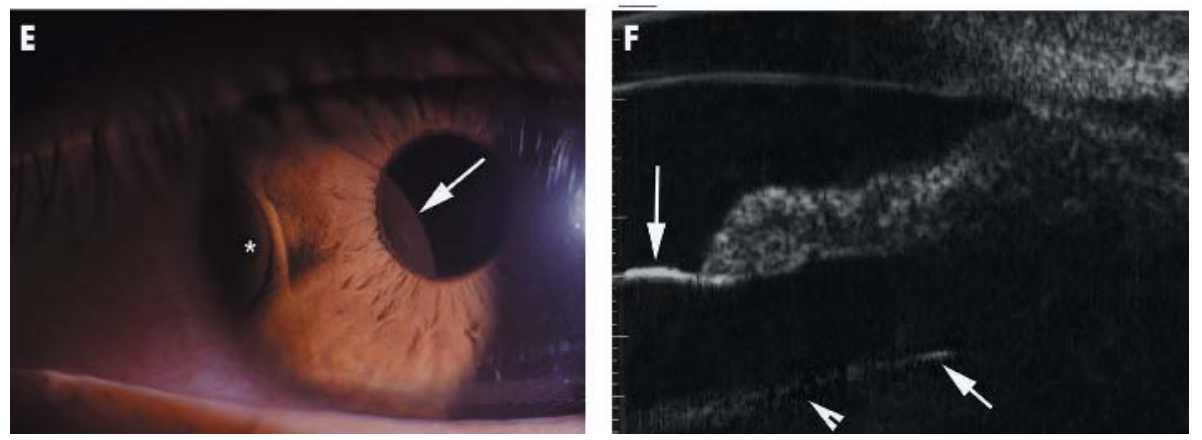

Table 1 Characteristics of patients evaluated for anterior segment lesions

\begin{tabular}{ll}
\hline Total patients & 130 \\
Total eyes & 132 \\
Sex & \\
Male & 51 \\
Female & 79 \\
Age range (years) & $8-87$ \\
Eye & \\
Right & 63 \\
Left & 65 \\
Bilateral & 2 \\
Location & 55 \\
Melanomas* ( $n=$ eyes) & 18 \\
Iris & 16 \\
Ciliary body & 21 \\
Anterior choroids & \\
Cysts ( $n=$ eyes) & 21 \\
Iridociliary & 26 \\
Naevus ( $n=$ eyes) & 6 \\
Iris & \\
Othert ( $n=$ eyes) & \\
Enucleation & \\
\hline &
\end{tabular}

$(\mathrm{n}=6)$ there was good correlation between UBM and histopathology defining disease extent.

Twenty one patients were referred with anterior choroidal tumours. All were pigmented and had B-scan features consistent with melanoma. ${ }^{6}$ UBM excluded CB involvement in 13 cases and confirmed involvement in eight (table 3). All were treated by PBT after tantalum marker clip placement. For all tumours with CB involvement, intraoperative UBM was used to evaluate margins.

Patients treated by PBT $(n=57)$ were followed at our institution (mean follow up 26 months; range 6-55 months). Follow up included 3-6 monthly examination, photography, fluorescein angiography, UBM, and A/B-scan. Three patients with CB melanoma and one with choroidal melanoma demonstrated marginal recurrence by UBM. In one patient treated elsewhere by brachytherapy, trans-scleral extension was detected. He refused enucleation and was re-treated by PBT. The remaining patients underwent secondary enucleation. Histopathology confirmed the UBM findings of incomplete treatment.

There was 29\% correspondence between anatomical structures involved by tumour identified by A/B-scan $v$ UBM. CB involvement was identified more frequently by UBM $v \mathrm{~A} / \mathrm{B}$-scan in iris melanoma $(\mathrm{p}<0.01)$ and choroidal melanoma $(p=0.02)$. Iris involvement was identified in eight cases of CB melanoma $v$ one case identified by A/B-scan $(\mathrm{p}=0.02)($ table 3$)$. 
Table 2 UBM features of most common anterior segment cystic and solid mass lesions encountered

\begin{tabular}{|c|c|c|c|}
\hline & & MLT (mm) & MBD (mm) \\
\hline Diagnosis & UBM features & Mean (range) & Mean (range) \\
\hline Primary neuroepithelial cyst & $\begin{array}{l}\text { Highly reflective, smooth cyst wall }<0.2 \mathrm{~mm} \text { thick } \\
\text { Anechoic centre } \\
\text { May be bilateral }(42 \%) \text { or multiloculated }(26 \%) \\
\text { No solid component* } \\
\text { Iris location: peripheral }(n=22) \text {, mid-zone }(n=6) \text { and central }(n=2) \text {. Usually } \\
\text { inferotemporal iris }\end{array}$ & ND & $1.7(0.8-3.5)$ \\
\hline Iris naevus & $\begin{array}{l}\text { Solid plaque-like lesion on the anterior iris surface and/or stroma } \\
\text { Heterogeneous internal reflectivity } \\
\text { Posterior iris surface flat or smoothly concave. } \\
\text { Minor degrees of pupil distortion ( } 19 \% \text { ). } \\
\text { Minimal or no growth tendency on serial biometry }\end{array}$ & $1.8(0.9-3.2)$ & $2.3(1.2-5.0)$ \\
\hline Iris melanoma & $\begin{array}{l}\text { Solid irregular iris mass } \\
\text { Variable internal reflectivity } \\
\text { Distortion of surrounding structures }(81 \%) \\
\text { Irregularity or convex bowing of posterior iris plane } \\
\text { Enlargement on serial biometry }\end{array}$ & $2.1(1.3-4.8)$ & $4.2(1.3-9.0)$ \\
\hline Ciliary body melanoma & $\begin{array}{l}\text { Oval shaped mass centred on the ciliary body } \\
\text { Usually solid, cavitation may occur }(9.5 \%) \\
\text { Low to medium internal reflectivity } \\
\text { Invasion of adjacent tissues may be evident }\end{array}$ & $6.3(1.7-10.3) \dagger$ & $8.9(1.7-17.9) \dagger$ \\
\hline Anterior choroidal melanoma & $\begin{array}{l}\text { Ciliary body /iris extension can currently be appreciated by UBM } \\
\text { Invasion usually appears as tissue of reduced reflectivity compared to the } \\
\text { surrounding ciliary body / iris. }\end{array}$ & ND & ND \\
\hline
\end{tabular}
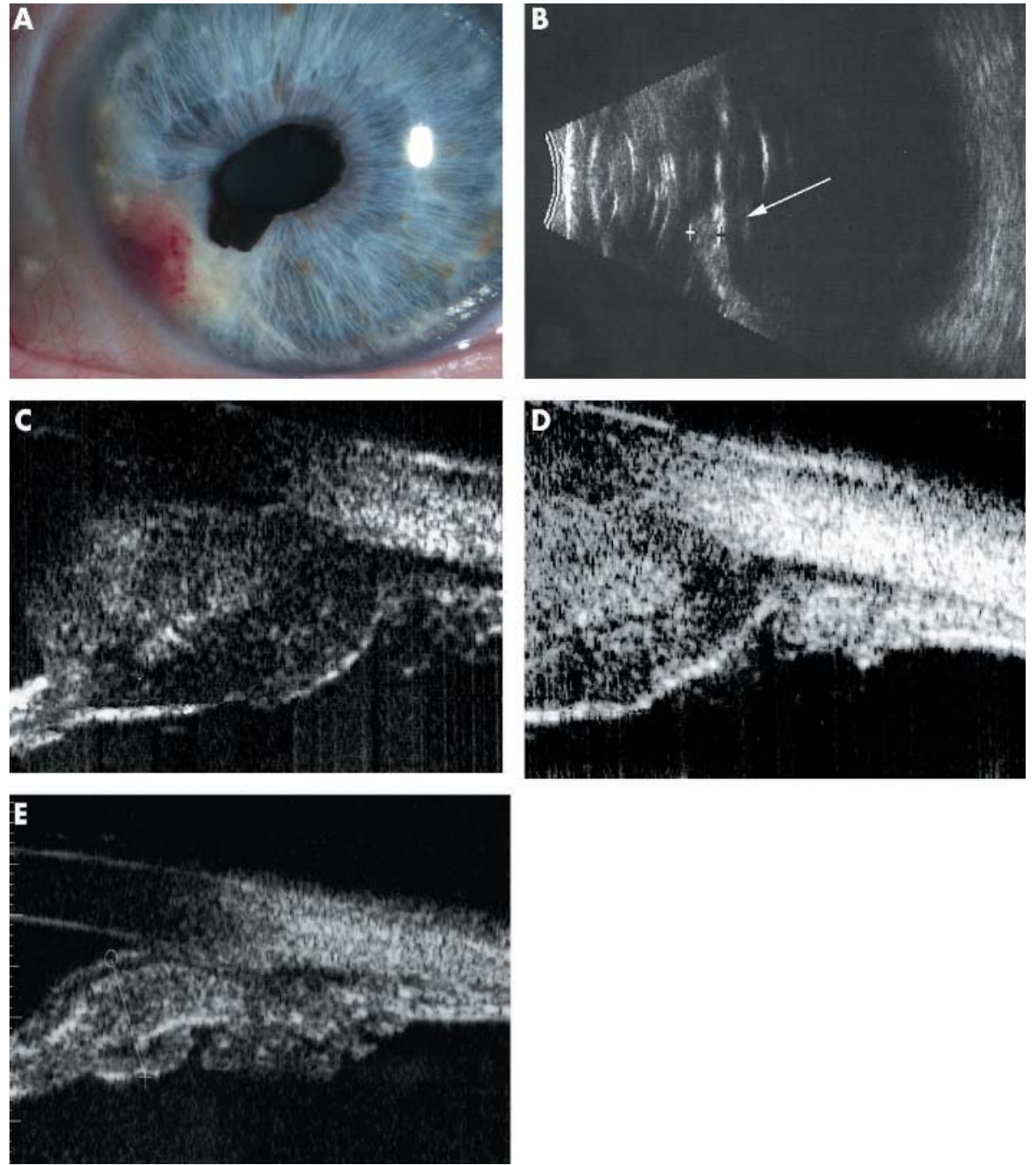

Figure 2 Iridociliary melanoma right eye (A-D). (A) Slit lamp photograph demonstrating an amelanotic mass with prominent intrinsic vasculature. This lesion showed growth tendency on serial UBM. Note marked pupil distortion. (B) B-scan shows a markedly thickened peripheral iris (arrow).

(C) UBM iris/iris root/ciliary body shows involvement by a solid mass with variable internal reflectivity. (D) UBM higher power. The mass invades the iris root and minimally involves the pars plicata of the ciliary body, evidenced by the area of reduced reflectivity compared to the surrounding tissues. UBM proved very useful

intraoperatively in defining the extent of ciliary body involvement in this case, as the amelanotic nature of the lesion precluded accurate assessment by transillumination. (E) Another patient with iris melanoma. Note distortion of the posterior iris plane by tumour. 


\begin{tabular}{|c|c|c|c|}
\hline Total melanomas (n) & 55 & & \\
\hline Evaluated by UBM (n) & 55 & & \\
\hline Evaluated by B-scan (n) & 45 & & \\
\hline Iris melanoma (n) & 14 & & \\
\hline Ciliary body melanoma (n) & 14 & & \\
\hline Choroidal melanoma (n) & 17 & & \\
\hline Number detected by B-scan or UBM & B-scan & UBM & p Value \\
\hline Ciliary body invasion by iris melanomas & 2 & 14 & $<0.01$ \\
\hline Ciliary body invasion by anterior choroidal melanomas & 2 & 8 & 0.02 \\
\hline Iris invasion by ciliary body /choroidal melanomas & 1 & 8 & 0.02 \\
\hline
\end{tabular}

\section{DISCUSSION}

The present study is the largest describing the use of UBM in consecutive ocular oncology patients. Most patients were referred by ophthalmologists who suspected the ocular lesion was a neoplasm. The high incidence of melanoma in our series $(42 \%)$ probably reflects this referral bias. Neuroepithelial cysts most commonly simulated iridociliary melanoma and UBM appearance was diagnostic in most cases.$^{7-10}$ However, the presence of a cyst does not exclude coexisting pathology. Identification of a solid $\mathrm{CB}$ mass associated with a thin walled cyst in one patient distinguished this mass as a melanoma (fig 1). This highlights the need for careful evaluation to exclude any solid component in an apparently benign cyst. Glaucoma, peripheral anterior synechiae, plateau iris syndrome, and corneal touch have been reported as complications of iridociliary cysts. ${ }^{79}$ In agreement with other series, we found these complications were uncommon and treatment rarely required. ${ }^{10}$

Documented growth is the most important indicator of malignancy in differentiating iris naevi from melanoma. ${ }^{511}$ UBM was an indispensable adjunct to photography for assessing growth, as both maximal thickness and diameter could be reliably measured, objectively validating this important feature. However, occasionally iris tumours displaying signs suggestive of malignancy have been reported to be histopathologically benign. ${ }^{11}$ Invasion of the CB typically
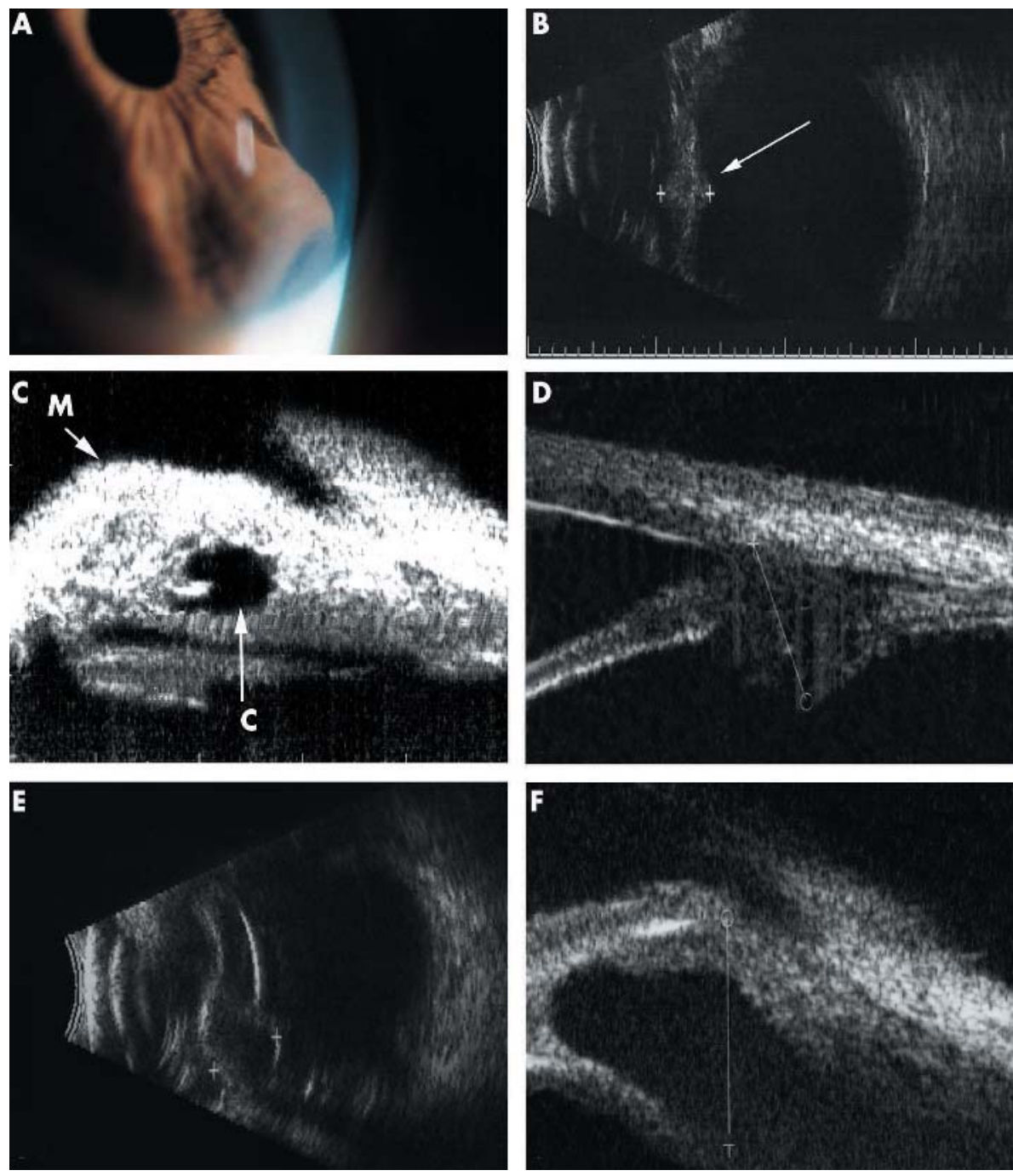

Figure 3 Iridociliary melanoma right eye. (A) Slit lamp photograph showing characteristic convex ciliary body tumour invading and displacing iris stroma. (B) B-scan ultrasound shows a solid mass with heterogeneous internal reflectivity. (C) UBM demonstrates that the tumour in (B), has internal cavitation ( $M$, melanoma; $C$, cavitation). (D) UBM, right eye, of a patient with a pigmented mass noted on routine glaucoma screening examination. The mass is solid and has low moderate internal reflectivity. The maximal diameter is $1.7 \mathrm{~mm}$. Note early invasion of the iris root and angle evidenced by reduced reflectivity compared to surrounding structures. (E) Ciliary body melanoma from another patient demonstrates prominent internal cavitation with pseudocyst formation. This lesion appeared to be cystic on conventional $B$-scan. (F) UBM of the patient in (E), however, revealed the solid component and thickened wall with medium reflectivity. 
appears on UBM as an area of reduced reflectivity in continuity with the iris mass, and is diagnostically important as naevi do not usually involve this structure (fig 2). ${ }^{611}$ Additionally, iris melanoma involvement of the iris root is an independent risk factor for metastasis. ${ }^{11}$ UBM of iris melanomas demonstrates a variable appearance, reflecting the clinical heterogeneity attributed to differences in lesion vascularity and cellularity. ${ }^{12}$ Reflecting the tendency for tissue distortion by melanomas, irregularity of the posterior iris surface was associated with most iris melanomas in this study, however was rarely observed in naevi (see fig 2E).

UBM is the only diagnostic tool which allows visualisation of small CB tumours. We were able to characterise and follow one patient with a CB tumour with a maximal diameter of $1.7 \mathrm{~mm}$ (fig 3D). Serial UBM demonstrated growth in this lesion including early invasion of the iris root. The main differential diagnoses include other pigmented CB tumours such as melanocytomas and pigment epithelial adenomas/ adenocarcinomas. ${ }^{13}$ These entities can be difficult to differentiate clinically and on UBM. All may appear as well defined CB masses with low to high internal reflectivity and invasive features. ${ }^{6}{ }^{13}$ In practice, many CB tumours remain undetected until they are relatively large in size, when they present symptomatically and require A/B-scan ultrasound to obtain accurate characterisation.

The estimated frequency of microscopic cavitation in uveal melanomas is $8.4 \% .^{14}{ }^{15}$ In rare instances, gross cavitation may occur, resulting in pseudocyst formation and confusion clinically and on A/B-scan with benign cysts. ${ }^{14}{ }^{15}$ We observed two CB melanomas with cavitation, one with pseudocyst formation (fig 3C, E, F). According to the largest reported series, compared to epithelial cysts, cavitating uveal melanomas tend to be larger (base: $8-15 \mathrm{~mm}$ /thickness: 2.2$14.1 \mathrm{~mm}$ ) and the pseudocyst wall is thicker (thinnest region: $0.4 \mathrm{~mm}$ ) with medium reflectivity. ${ }^{14}$ UBM analysis of our patient allowed the solid component and irregularly thickened wall to be appreciated.

Ring melanomas often present atypically with glaucoma, retinal dialysis, or heterochromia. ${ }^{16}$ A discrete mass is frequently not appreciated on examination, leading to delayed diagnosis. ${ }^{16}$ Tumour thickness can vary at different $\mathrm{CB}$ locations, causing the ring configuration of the tumour to be difficult to appreciate by A/B-scan, especially if the tumour is $<4 \mathrm{~mm}$ thick. As enucleation rather than conservative treatment is the management of choice for ring melanoma, we routinely survey the entire CB by sequential UBM clock hour imaging in cases of suspected iris or CB melanoma, or in cases where an underlying ring could be responsible for other atypical ocular findings. ${ }^{16}$

Precise definition of tumour margins is crucial for successful conservative management of anterior UM. ${ }^{517}$ Improved tumour localisation allows tighter therapeutic margins, reducing radiation exposure to surrounding tissues and potentially reducing treatment side effects. Intraoperative assessment of the extent of $\mathrm{CB}$ involvement by UM is particularly challenging. Transillumination can provide useful information; however, tumour melanin content, haemorrhage, and the degree of CB pigmentation can lead to inaccuracies. The present study demonstrates that UBM identifies $\mathrm{CB}$ involvement by adjacent melanomas significantly more often than A/B ultrasound. The accuracy of UBM in defining tumour margins is supported by studies which have demonstrated a high correlation (approximately 70\%) between margins in the iris and $\mathrm{CB}$ defined by UBM compared to histopathological margins on enucleated specimens.. ${ }^{418} 19$ In cases where UBM did not correlate with histopathological margins, UBM tended to overestimate the anterior tumour margin. ${ }^{4}$ We have found UBM very useful in defining anterior tumour margins and now use it as a routine intraoperative tool.

Fifty seven patients were followed after PBT. In four cases we found evidence of re-growth, the remaining tumours displaying stable or decreased dimensions. All were $\mathrm{CB}$ tumours that had been treated before our current practice of using UBM intraoperatively. In one case we were able to completely define the extent of trans-scleral recurrence with intraoperative UBM and retreat with PBT and scleral patch graft. This patient remains in complete remission with stable best corrected vision of 20/60 at 55 months follow up.

A/B-scan ultrasonography remains an essential component in evaluating tumours for posterior extension and larger melanomas that cannot be fully evaluated by UBM. A/B-scan can also be used to demonstrate features characteristic of melanoma including low internal reflectivity, internal acoustic hollowness and sound attenuation, choroidal excavation, orbital shadowing and the presence of spontaneous vascular pulsations. ${ }^{6}$ In some instances, the internal acoustic pattern obtained by conventional ultrasound will allow differentiation of the lesion (for example, melanoma $v$ haemangioma) not possible with UBM. ${ }^{6}$ There may be limitations to the use of UBM based on availability and user familiarity and, in these instances, A/B-scan may be of more use when correlated with clinical data.

In conclusion, UBM is very useful for anterior segment tumour management. The ability to characterise cystic lesions is diagnostic in most cases. UBM also allows reliable biometry, providing objective assessment of lesion size. When serial observation is indicated, UBM can detect growth with greater precision. Finally, UBM is helpful in treatment planning, particularly in establishing anterior margins.

\section{ACKNOWLEDGEMENTS}

This study was supported in part by Knights Templar Eye Foundation, Inc, Chicago, IL (JMO), Fight For Sight, Inc, New York, NY (JMO), That Man May See Inc, San Francisco, CA (JMO/ RMC), Physician Scientist Award, Research to Prevent Blindness, New York, NY (JMO), R01 EY13812 Research Grant, National Eye Institute, Bethesda, MD (JMO), UCSF Department of Ophthalmology Core Grant EY02162, National Eye Institute, Bethesda, MD (JMO), and The National Health and Medical Research Council, Australia (RMC).

\section{Authors' affiliations \\ R M Conway, T Chew, P Golchet, K Desai, S Lin, J O'Brien, Department of Ophthalmology, University of California, San Francisco, CA, USA R M Conway, Sydney Eye Hospital and Save Sight Institute, University of Sydney, NSW, Australia}

Ethical approval: The current study was carried out with Institutional Review Board approval of the University of California San Francisco and according the tenets of the Declaration of Helsinki.

Correspondence to: R Max Conway, Sydney Eye Hospital and Save Sight Institute, University of Sydney, NSW, Australia; rmaxconway@ hotmail.com

Accepted for publication 9 December 2004

\section{REFERENCES}

1 Pavlin CJ, McWhae JA, McGowan HD, et al. Ultrasound biomicroscopy of anterior segment tumors. Ophthalmology 1992;99:1220-8.

2 Finger PT. Plaque radiation therapy for malignant melanoma of the iris and ciliary body. Am J Ophthalmol 2001;132:328.

3 Katz NR, Finger PT, McCormick SA. Ultrasound biomicroscopy in the management of malignant melanoma of the iris. Arch Ophthalmol 1995; 113:1462-3.

4 Marigo FA, Finger PT, McCormick SA. Iris and ciliary body melanomas: ultrasound biomicroscopy with histopathologic correlation. Arch Ophthalmol 2000;118:1515-21

5 Marigo FA, Finger PT. Anterior segment tumors: current concepts and innovations. Surv Ophthalmol 2003;48:569-93. 
6 Pavlin CJ, Foster FS. Ultrasound biomicroscopy of the eye. In: Byrne, Green, eds. Ultrasound of the eye and orbit, 2nd ed. St Louis: Mosby, 223-43.

7 Marigo FA, Esaki K, Finger PT, et al. Differential diagnosis of anterior segment cysts by ultrasound biomicroscopy. Ophthalmology $1999 \cdot 106 \cdot 2131-5$.

8 Shields JA, Sanborn GE, Augsburger JJ. The differential diagnosis of malignant melanoma of the iris. A clinical study of 200 patients. Ophthalmology 1983;90:716-20

9 Augsburger JJ, Affel LL, Benarosh DA. Ultrasound biomicroscopy of cystic lesions of the iris and ciliary body. Trans Am Ophthalmol Soc 1996;94:259-71.

10 Lois N, Shields CL, Shields JA, et al. Primary cysts of the iris pigment epithelium. Ophthalmology 1998;105:1879-85.

11 Shields CL, Shields JA, Materin M, et al. Iris melanoma: risk factors for metastasis in 169 consecutive patients. Ophthalmology $2001 ; 108: 172-8$

12 Fuller DG, Snyder WB, Hutton WL, et al. Ultrasonographic features of choroidal malignant melanomas. Arch Ophthalmol 1979:97:1465-72.
13 LoRusso FJ, Boniuk M Font RL. Melanocytoma (magnocellular nevus) of the ciliary body: report of 10 cases and review of the literature. Ophthalmology 2000; 107:795-800

14 Lois N, Shields CL, Shields JA, et al. Cavitary melanoma of the ciliary body. A study of eight cases. Ophthalmology 1998;105:1091-8.

15 Kennedy RE. Cystic malignant melanomas of the uveal tract. Am J Ophthalmol 1948;31:159-67.

16 Demirci H, Shields CL, Shields JA, et al. Ring melanoma of the ciliary body: report on twenty-three patients. Retina 2002;22:698-706.

17 Conway RM, Chua WC, Qureshi C, et al. Primary iris melanoma: diagnostic features and outcome of conservative surgical treatment. $\mathrm{Br} J$ Ophthalmol 2001;85:848-54.

18 Nordlund JR, Robertson DM, Herman DC. Ultrasound biomicroscopy in management of malignant iris melanoma. Arch Ophthalmol 2003;121:725-7.

19 Maberly DA, Pavlin CJ, McGowan HD, et al. Ultrasound biomicroscopic imaging of the anterior aspect of peripheral choroidal melanomas. Am J Ophthalmol 1997;123:506-14.

\section{bmjupdates+}

bmjupdates+ is a unique and free alerting service, designed to keep you up to date with the medical literature that is truly important to your practice.

bmjupdates+ will alert you to important new research and will provide you with the best new evidence concerning important advances in health care, tailored to your medical interests and time demands.

Where does the information come from?

bmjupdates+ applies an expert critical appraisal filter to over 100 top medical journals A panel of over 2000 physicians find the few 'must read' studies for each area of clinical interest

Sign up to receive your tailored email alerts, searching access and more...

www.bmjupdates.com 